OPEN ACCESS

Edited by:

Luca Domenico D'Andrea, National Research Council (CNR), Italy

Reviewed by: Helmut Bergler,

University of Graz, Austria Xiyuan Yao,

The Scripps Research Institute, United States

*Correspondence: Jerónimo Bravo jbravo@ibv.csic.es

Specialty section:

This article was submitted to

Molecular Recognition,

a section of the journal

Frontiers in Molecular Biosciences

Received: 01 June 2021

Accepted: 20 July 2021

Published: 01 September 2021

Citation:

Orea-Ordóñez L, Masiá S and Bravo J (2021) Peptides Targeting the Interaction Between Erb1 and Ytm1

Ribosome Assembly Factors. Front. Mol. Biosci. 8:718941. doi: $10.3389 /$ fmolb.2021.718941

\section{Peptides Targeting the Interaction Between Erb1 and Ytm1 Ribosome Assembly Factors}

\author{
Lidia Orea-Ordóñez, Susana Masiá and Jerónimo Bravo*
}

Department Genomics and Proteomics, Instituto de Biomedicina de Valencia, Spanish National Research Council (CSIC), Valencia, Spain

Ribosome biogenesis is an emerging therapeutic target. It has been proposed that cancer cells are addicted to ribosome production which is therefore considered a druggable pathway in cancer therapy. Cancer cells have been shown to be more sensitive to inhibition of the ribosome production than healthy cells. Initial attempts of inhibiting ribosome biogenesis have been focused on the inhibition of transcription by targeting RNA Pol I. Despite being a promising field of research, several limitations have been identified during the development of RNA Pol I inhibitors, like the lack of specificity or acquired resistance. Ribosome biogenesis is a multistep process and additional points of intervention, downstream the very initial stage, could be investigated. Eukaryotic ribosome maturation involves the participation of more than 200 essential assembly factors that will not be part of the final mature ribosome and frequently require protein-protein interactions to exert their biological action. Using mutagenesis, we have previously shown that alteration of the complex interface between assembly factors impairs proper ribosome maturation in yeast. As a first step toward the developing of ribosome biogenesis inhibitory tools, we have used our previously solved crystal structure of the Chaetomium thermophilum complex between the assembly factors Erb1 and Ytm1 to perform a structure-guided selection of interference peptides. The peptides have been assayed in vitro for their ability to bind their cellular partner using biophysical techniques.

Keywords: structural-guided peptide selection, interference peptides, targeting ribosome biogenesis, protein-protein interactions, Erb1/Ytm1 complex

\section{INTRODUCTION}

The synthesis of ribosomes in eukaryotes is a sophisticated and energy-demanding process requiring the participation of more than 200 assembly factors that will not be part of the final mature ribosome although are required for a correct ribosome biogenesis. The initial steps of ribosome assembly take place in the nucleolus where rRNA is transcribed by RNA polymerase I (RNA pol I) [reviewed in (Baßler and Hurt, 2019)] under the regulation of both tumor suppressor genes (including p53, Rb, and Arf) and oncogenes (including MYC, MAPK/ERK, PI3K, and AKT) (Montanaro et al., 2008; Morcelle et al., 2019). Increased ribosome biogenesis is important for cell transformation or tumorigenesis and it is assumed as a general trend in cancer cells that need to make extra ribosomes in order to produce more proteins to sustain uncontrolled cell division (Orsolic et al., 2016) (Truitt and Ruggero, 2016). Interestingly, it has been shown that cell proliferation can be 
blocked by inhibiting the production of new ribosomes since impaired ribosome biogenesis induces a checkpoint control that prevents cell cycle progression (Volarević et al., 2000). It is therefore not surprising that biogenesis of the ribosomes has been accumulating growing attention as a potential new therapeutic target.

Initial attempts to specifically target ribosome biogenesis have been focused on the downregulation of RNA pol I. Several recently described small molecules like CX-5461, CX-3543, BMH-21, or CID-765471 are now providing evidence that inhibition of ribosome biogenesis by targeting transcription of ribosomal DNA has a promising therapeutic potential (Bywater et al., 2012; Colis et al., 2014). However, lack of specificity and acquired resistance suggest that a new generation of Pol I inhibitors should be developed. Moreover, the only inhibitor that reached clinical trials has shown additional activities contributing to its toxicity profile and resistance, and therefore, additional points of intervention during the ribosome maturation process should be explored apart from the inhibition of transcription (Catez et al., 2019; Ferreira et al., 2020). Despite the fact that the complexity of the process yields a large repertoire of potential targets, only very few chemical inhibitors of ribosome biogenesis are known so far (Awad et al., 2019). Apart from the lack of molecular details in the process, the main reason for this limited number of inhibitors is that the complex ribosome biogenesis pathway is orchestrated by a wide range of macromolecular interactions sequentially coordinated to promote the correct ribosome maturation. Ribosome assembly factors frequently require protein-protein interactions in order to exert their biological actions ( $\mathrm{Li}$ et al., 2017) and these have been traditionally hard to target by small molecule drugs given the absence of grooves or binding pockets in the interaction surface.

Nop7, Erb1, and Ytm1 are assembly factors that form a discrete heterotrimer that can be detected in isolation from the pre-ribosomal particles (Tang et al., 2008). The so-called Nop7 complex (PeBoW in mammals) is essential for a correct maturation of the ribosomal $60 \mathrm{~S}$ subunit. The three components guarantee the correct maturation of 5' end of 5.8S rRNA, thus facilitating its association with $25 \mathrm{~S}$ rRNA in the mature ribosome (Granneman et al., 2011). We have previously reported the structural resolution of the Chaetomium thermophilum Erb1/Ytm1 complex (Marcin et al., 2015; Wegrecki et al., 2015). The crystal structure shows Ytm1 bound to the carboxy-terminal portion of Erb1. Integrity of the heterotrimer assembly is essential for exerting its biological action. In fact, we previously showed that compromising the stability of the Erb1/Ytm1 interaction has an effect on proliferation in yeast (Wegrecki et al., 2015).

Using our previous Erb1/Ytm1 structure, we have performed a structure-guided selection of a set of peptides derived from their sequences and test their ability to bind to their respective cellular partners, Erb1 or Ytm1. Our results open the possibility to obtain peptides with the ability to interfere in the ribosome biogenesis pathway. To our knowledge, this is the first report of peptides designed to target ribosome biogenesis.

\section{MATERIALS AND METHODS}

\section{Cloning, Expression, and Purification of Ytm1 and Erb1}

Protein expression and purification was carried out following the protocol described in a study by Wegrecki et al. (2015). The YTM1 gene from Chaetomium thermophilum was obtained by total cDNA amplification and cloning in pOPIN-F using an InFusion cloning system commercial kit (Clontech). Sf9 insect cells were grown in the Sf900 II SFM medium (Gibco) and were transfected with Ytm1-pOPINF and linearized Ian Jones bacmid (Zhao et al., 2003). Baculovirus generated were amplified and used to induce protein expression for $72 \mathrm{~h}$ at $27^{\circ} \mathrm{C}$. Erbl was cloned in a pET28-NKI/LIC 6His/3C vector (from NKI Protein Facility, Amsterdam) by the ligase independent cloning (LIC) method and expressed in Escherichia coli (DE3) BL21 CodonPlus (RIPL) using an auto-induction system (Studier, 2005). The $6 \mathrm{xHis}$ tagged Ytm 1 and Erb1 proteins were purified using a Histrap-HP Ni charged column (GE Healthcare) eluting with a 20-500 mM imidazole gradient. An additional step of a HiTrap Heparin HD column (GE Healthcare) eluting with $0.2-1.5 \mathrm{M}$ $\mathrm{NaCl}$ gradient was included for Erb1. A final polish step of size exclusion chromatography (HiLoad 16/60 Superdex 200 column, GE Healthcare) was performed for both proteins that were flash cooled under liquid nitrogen and stored at $-80^{\circ} \mathrm{C}$ until use.

\section{Biolayer Interferometry Assays}

Peptides were commercially obtained from Synpeptide Co., Ltd., Shanghai, China. All experiments have been made in triplicates and include control curves for bait and analyte only.

\section{Peptide Interference Experiments}

Potential interference peptide samples were evaluated by their ability to interfere the kinetics and affinity of the complex between Erb1 and Ytm1 as measured by Biolayer Interferometry (BLItz, Pall FortéBio Corp.) using Ni-NTA biosensors (FortéBio) and $50 \mathrm{mM}$ HEPES, pH 7.5; $150 \mathrm{mM}$ $\mathrm{NaCl} ; 5 \%(\mathrm{v} / \mathrm{v})$ glycerol; and $2 \mathrm{mM} \beta$-mercaptoethanol (BME) buffer. To set the reference $\mathrm{K}_{\mathrm{D}}$, an $80 \mu \mathrm{g} / \mathrm{ml}$ solution of Erb1 was immobilized to the biosensor in order to obtain binding curves for increasing concentrations of Ytm1. According to this, an evaluation of Erb1-derived peptides (P1-P3) was performed using $5 \mu \mathrm{M}$ Ytm1 previously incubated during $30 \mathrm{~min}$ at equimolar concentrations of each peptide. New $K_{D}$ values were determined. Similarly, an $80 \mu \mathrm{g} / \mathrm{ml}$ solution of Ytm1 was immobilized to the biosensor in order to obtain binding curves for increasing concentrations of Erb1. $20 \mu \mathrm{M}$ Erb1concentration was selected for the analyte. Each Ytm1-derived peptide (P4-P6) was evaluated by preincubation of equimolar concentrations with $20 \mu \mathrm{M}$ Erb1 assayed against immobilized Ytm1 of $80 \mu \mathrm{g} / \mathrm{ml}$.

\section{Peptide Affinity Experiments}

BLI was used to determine the affinity between Ytm1 and biotinylated peptides using streptavidin biosensors (FortéBio). The biosensors were hydrated $10 \mathrm{~min}$ in the same buffer used on the interference assay, with $0.05 \%(\mathrm{w} / \mathrm{v})$ bovine serum albumin 
(BSA). $50 \mu \mathrm{g} / \mathrm{ml}$ of biotinylated peptide was immobilized at the streptavidin biosensor. Increasing concentrations of Ytm1 were used to calculate the $\mathrm{K}_{\mathrm{D}}$ values using the Blitz Pro 1.2 software using the implemented equations for association and dissociation:

Association phase:

$$
\mathrm{y}=\mathrm{R}_{\max } \frac{1}{1+\frac{k_{\mathrm{d}}}{k_{a} *[\text { Analyte }]}}\left(1-e^{-\left(k_{\mathrm{a}} *[\text { Analyte }]+k_{\mathrm{d}}\right) \mathrm{x}}\right) .
$$

Dissociation phase:

$$
\begin{gathered}
\mathrm{y}=\mathrm{y}_{0} e^{-k_{\mathrm{d}}\left(\mathrm{x}-\mathrm{x}_{0}\right)} . \\
\mathrm{y} 0=\mathrm{R}_{\max } \frac{1}{1+\frac{k_{\mathrm{d}}}{k_{a} *[\text { Analyte }]}}\left(1-e^{-\left(k_{\mathrm{a}} *[\text { Analyte }]+k_{\mathrm{d}}\right) \mathrm{x}_{0}}\right) .
\end{gathered}
$$

\section{Differential Scanning Fluorimetry}

Thermofluor assays (Pantoliano et al., 2001) were performed on a 7,500 Fast Real-Time PCR System (Applied Biosystems) measuring the gradual fluorescence generated by a 1:1,000 dilution of SYPRO Orange Protein Stain Gel (Supelco, MerckSigma) along with $1^{\circ} \mathrm{C} / \mathrm{min}$ temperature increase from $20^{\circ} \mathrm{C}$ until $85^{\circ} \mathrm{C}$.

The samples were mixed containing the protein Ytm1 at $5 \mu \mathrm{M}$ in $50 \mathrm{mM}$ HEPES pH 7.5, $150 \mathrm{mM} \mathrm{NaCl}$ and peptides P1-P3 at $1 \mathrm{mM}$ concentration. Previously, lyophilized P2 and P3 were resuspended in the same Ytm1 buffer and P1 was solubilized in $10 \mathrm{mM}$ Tris (hydroxymethyl)-methylamine (Tris-HCl), pH 8.8 for P1. Data of triplicated experiments were analyzed using GraphPad Prism 5.01 software.

\section{MicroScale Thermophoresis}

Microscale thermophoresis (MST) was used to determine the binding affinity between Ytm1 and P1. Ytm1 was labeled with a Red-NHS second-generation dye kit $(100 \mu \mathrm{l}$ at $10 \mu \mathrm{M}$ of protein $+300 \mu \mathrm{M}$ dye solution) for $30 \mathrm{~min}$ at room temperature in the dark. $5 \mathrm{nM}$ of labeled Ytm 1 in $50 \mathrm{mM}$ HEPES pH 7.5, NaCl $150 \mathrm{mM}$ supplemented with $0.1 \%$ (v/v) Pluronic F-127 was used for the assay. P1 stock is diluted in $10 \mathrm{mM}$ Tris (hydroxymethyl)methylamine (Tris-HCl), $\mathrm{pH}$ 8.8. Ytm1 and $\mathrm{P} 1$ were mixed at 1:1 molar ratio in sixteen serial dilutions $(1,230 \mu \mathrm{M}-0.0751 \mu \mathrm{M})$ using the same buffer. Measurements were taken on a Monolith NT.115 instrument (NanoTemper Technologies). Similarly biding between Ytm1 and P3 using MST was tested using the same Ytm1 buffer also for P3. Curve fitting and $\mathrm{K}_{\mathrm{D}}$ calculations every $10 \mathrm{~min}$ were analyzed from three independent experiments with MO. Maximum binding was observed at $50 \mathrm{~min}$. Curves were analyzed using Affinity Analysis Software (NanoTemper Technologies).

\section{Bioinformatics}

Buried surface calculations were performed using the protein interfaces, surfaces, and assemblies service PISA at the European Bioinformatics Institute (http://www.ebi.ac.uk/ pdbe/prot_int/pistart.html) (Krissinel and Henrick 2007) using $\mathrm{PDB}$ ID $5 \mathrm{cxb}$ and in silico alanine scanning of the interaction surface was performed using the DrugScorePPI Web Interface (https://cpclab.uni-duesseldorf.de/dsppi/) (Krüger and Gohlke, 2010) only considering polypeptide atoms. $\Delta G$ computed for alanine mutants for each $5 \mathrm{cxb}$ polypeptide chain is compared to $\Delta G$ computed from the wild type complex. Resulting $\Delta \Delta \mathrm{G}$ predicts the contribution of a given side chain to the wild type complex stability $(\Delta \Delta \mathrm{G}=$ $\Delta$ GALAcomplex $-\Delta$ GWTcomplex). Interacting atoms between Erb1 and Ytm 1 were also analyzed using the $5 \mathrm{cxb}$ coordinates program contact in the CCP4 suite (Winn et al., 2011). Molecular graphics in Figure 1 were performed with UCSF Chimera (Pettersen et al., 2004).

\section{RESULTS}

\section{Interaction Surface Analysis and Peptide Selection}

Our Erb1/Ytm1 heterodimer crystal structure (PDB ID 5CXB) (Wegrecki et al., 2015) shows that the central part of the $\beta$-propeller of Ytm1 provides a large docking surface for the bottom face of blades 1, 2, and 7 from Erb1 that is additionally held in place by two lateral extensions from Ytm1. The Ytm1 $\beta$-propeller top face, away from the N-terminal Ubiquitin-like domain, establishes extensive contacts with the side face of Erb1 C-terminal $\beta$-propeller (Figure 1A) with a predominant role of blade 7 . The Erb1/Ytm1 heterodimer is mainly maintained by electrostatic forces with some hydrophobic regions also involved in the interaction (Figure 1B). No clear grooves have been detected in the heterodimer interaction surface.

Manual inspection of the interaction surface between Erb1 and Ytm 1 combined with buried surface area upon binding calculations using PISA (Krissinel and Henrick, 2007), together with an analysis of the interactions and an alanine scanning of the interacting residues to locate hotspots using DrugScore $^{\text {PPI }}$ (Krüger and Gohlke, 2010), revealed several areas of interaction (Figure 1C, Supplementary Table S1, S2). The last $\beta$-strand (" $1 \mathrm{~d}$ ") of blade 7 in Erb1 contacts loop " $6 \mathrm{~d}-6 \mathrm{a}$ " and a long extension that appears between strands " $7 \mathrm{~d}$ " and " $7 \mathrm{a}$ " of Ytm1 (the knob formed by residues 444-460). A second interaction area involves the entrance of the central tunnel of Ytm1 as a docking site for a loop between strands "1c-2d" from the first blade of Erb1 (481-486) (Figure 1C). The loop contains three well conserved residues: E481, T484, and R486 that establish a network of electrostatic interactions with also conserved amino acids from blades 1, 2, 3, and 7 of Ytm1. We have previously reported the relevance of the second area of interaction since the $\mathrm{R} 486 \mathrm{E}$ point mutation decreased the affinity of the interaction by two orders of magnitude without affecting structural integrity. The equivalent mutation in yeast impaired growth in yeast and affected 60S subunit biogenesis (Wegrecki et al., 2015). As indicated by the in silico alanine scanning analysis, this interacting region also suggests a hot spot between Erb1 and Ytm1 (Figure 1C, Supplementary Table S1). Erb1 a-b 
A

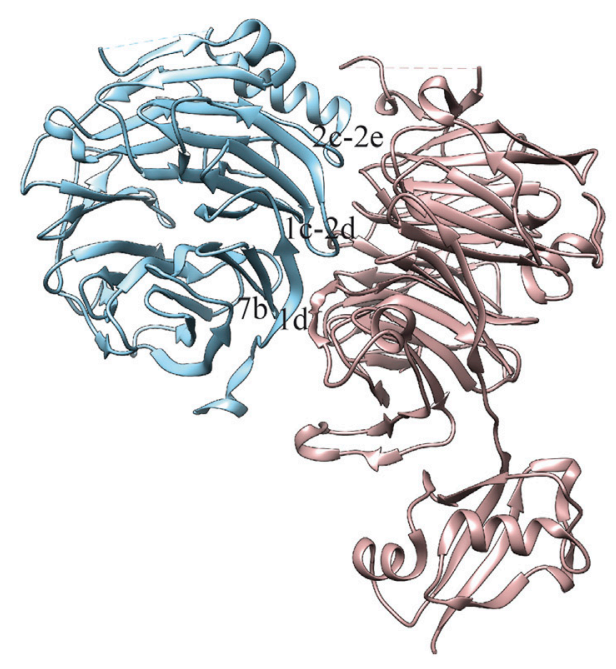

C

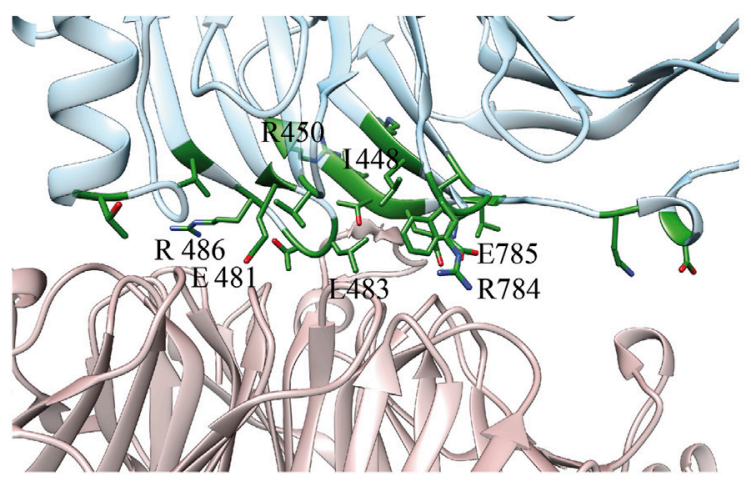

B

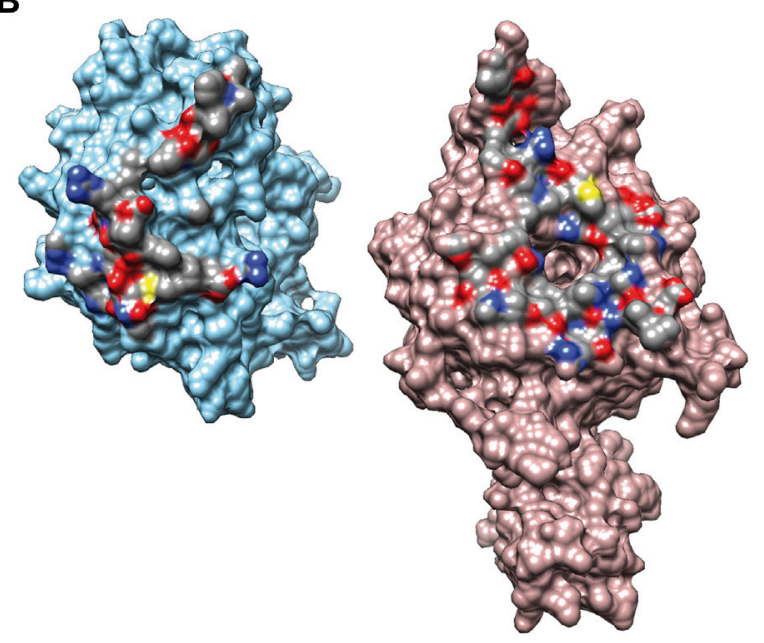

D

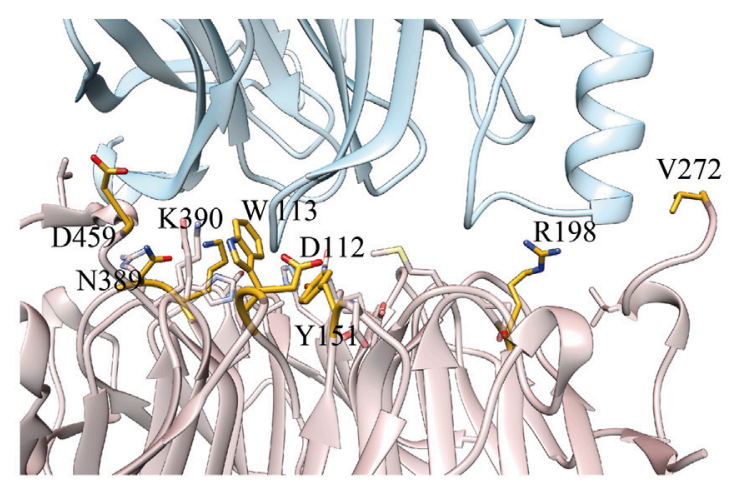

E

$\mathbf{F}$
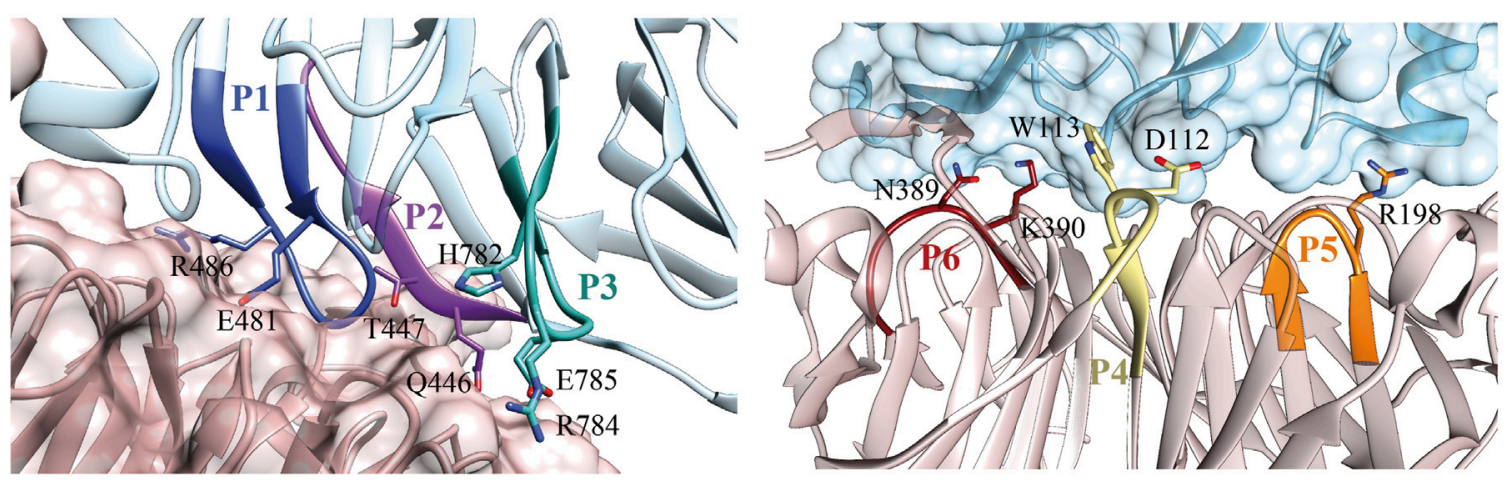

FIGURE 1 | Erb1/ $\mathrm{tm} 1$ interaction. (A) Ribbon representation of the heterodimer. Top face of Ytm1 $\beta$-propeller in pale pink interacts with Erb1 $\beta$-propeller's side in blue. The main secondary structure-interacting motifs are shown in Erb1 (B) Surface representation of the individual components with the interaction areas facing toward the observer. Atoms from residues involved in the interaction are colored by atom, carbon in grey, oxygen in red, and nitrogen in blue. The rest of the surface is colored in blue for Erb1 and pale pink for Ytm1. (C) Calculated Erb1 $\Delta \Delta G$ values from in silico alanine scan. Residues from Erb1 with calculated $\Delta \Delta G>0.5 \mathrm{kcal} / \mathrm{mol}$ are depicted in green. (D) Calculated $\mathrm{Ytm} 1 \Delta \Delta \mathrm{G}$ values from in silico alanine scan. Ytm1 residues with calculated $\Delta \Delta \mathrm{G}>1.0 \mathrm{kcal} / \mathrm{mol}$ are represented in yellow. (E) Selected peptides derived from Erb1. Each peptide is colored with a different shade of blue and is labeled according to Table 1. Side chains of interacting residues establishing electrostatic interactions are shown. (F) Selected peptides derived from Ytm1. The three selected peptides are depicted in brown, yellow, and orange for P6, P4, and P5, respectively (Table 1). Side chains of interacting residues establishing electrostatic interactions are shown. 
TABLE 1 | Peptides used in this study. Residue numbering is referred to the Chaetomium thermophilum sequences.

\begin{tabular}{llll}
\hline Peptide & \multicolumn{1}{c}{ Sequence } & Protein & Residues \\
\hline P1 & WWELLTGRQW & Erb1 & $479-489$ \\
P2 & QQTIFRGH & Erb1 & $445-452$ \\
P3 & DWHPREPWCV & Erb1 & $780-789$ \\
P4 & HDDWVSA & Ytm1 & $110-116$ \\
P5 & AGMDRTV & Ytm1 & $194-200$ \\
P6 & RGHANKV & Ytm1 & $385-391$ \\
\hline Biot-P1 & Biotin-WWELLTGRQWW & Erb1 & $479-489$ \\
Biot-P3 & Biotin-DWHPREPWCV & Erb1 & $780-789$ \\
Biot-P6 & Biotin-RGHANKV & Ytm1 & $385-391$ \\
\hline
\end{tabular}

loop from blade 7 participates in another interaction area with Ytm 1 loops from blade 3. Finally an insertion in blade 2 of the $\beta$-propeller of Erb1 between $2 c$ and 2e (Supplementary Figure S1) interacts with loops from blades 2 and 3 and an extension between strands $3 \mathrm{c}$ and $4 \mathrm{~d}$ from Ytm 1 (Supplementary Figure S2). This Erb1 insertion area shows poor sequence conservation (Supplementary Figure S1) and will not be taken it into further consideration. Ytm1 residues interacting with Erb1 appear to cluster mostly on one side of the interaction surface (Figure 1D) although they do not show sequence continuity of interacting residues (Figure 1D, Supplementary Figure S2 and Supplementary Table S2).

According to the previous observations, a set of six peptides, summarized in Table 1, were selected derived from Erb1 sequence and Ytm1 (Figures 1E,F and Supplementary Figures S1, S2).

\section{Peptide Competition Assay}

Biolayer interferometry interference assays were used to evaluate the ability of each peptide to interfere in the in vitro formation of the Erb1/Ytm1 complex. Erb1 was immobilized to a Ni-NTA biosensor and Ytm1 was used as analyte for testing direct interaction. The binding affinity is $3.2 \mathrm{e}-8 \mathrm{M}$ (Supplementary Table S3), similar to the previously reported values using microcalorimetry (Wegrecki et al., 2015). Peptides were evaluated for their ability to decrease in the Erb1/Ytm1 complex formation by preincubating the analyte with each peptide derived from the immobilized partner. Figures 2A,B show typical curves from the interference assay using biolayer interferometry. The black line depicts the reference curve obtained in the absence of peptide preincubation. Erb1-derived peptides were evaluated by their ability to decrease Erb1/Ytm1 complex affinity. Each P1-3 peptide was preincubated for half an hour at $4^{\circ} \mathrm{C}$ with an equimolar concentration of Ytm1 used as an analyte for binding with Erb1, previously immobilized to the NiNTA biosensor (Figure 2A). Peptides P1 and P3 showed a decrease of more than one order of magnitude (1.9 and 1.7e$7 \mathrm{M}$ respectively) with respect the Erb1/Ytm1 binding curve, in the absence of peptide. Similarly, an assay immobilizing Ytm1 to the Ni-NTA biosensor was used with Erb1 as analyte preincubated at equimolar concentrations with P4-6 Ytm1derived peptides. Only peptide P6 showed a significant decrease $(2.4 \mathrm{e}-6 \mathrm{M})$ with respect to the reference Ytm1/Erb1 binding curve (Figure 2B).

\section{Direct Peptide Binding Assays}

According to P1, P3, and P6 results, a new N-terminal biotynilated peptide synthesis was ordered using the same sequences (Biot-P1, Biot-P3, and Biot-P6, Table 1). Biotynilated peptides Biot-P1 and Biot-P3 were immobilized to the streptavidin biosensor and Ytm1 binding was measured. Similarly, Biot-P6 was immobilized to the streptavidin biosensor followed by Erb1 binding. As indicated in Figure 2C, we could not detect binding for Biot-P6/Erb1. Peptides Biot-P1 and Biot-P3 showed an affinity for Ytm1 in the $\mathrm{mM}$ range (Figures 2D,E). To further confirm the interaction a differential scanning fluorimetry assay was performed. A thermofluor assay of Ytm1 on its own and in the presence of $1 \mathrm{mM}$ P3 did not show any significant thermal shift (Figure 2F) Similarly, no significant thermal shift in Ytm1 was observed in the presence of $1 \mathrm{mM} \mathrm{P} 2$ (Supplementary Figure S3). A shift of several degrees in the Tm was observed in the presence of $1 \mathrm{mM} \mathrm{P1}$ indicating binding with Ytm1 (Figure 2G). Inconsistent results were obtained in DSF using Erb1 and a proper Tm could not be obtained so thermal shift could not be evaluated for P6. To further demonstrate the ability of P1 to interact with Ytm1, the binding was also monitored in free solution by the change in the thermophoresis of Ytm1 upon interaction with P1. Binding affinity determined using microscale thermophoresis showed a Kd in the mM range (8.2e-6M, Supplementary Table S3) consistent with affinities observed using interferometry and further confirming a direct interaction between P1 and Ytm1 (Figure 2H). Experimental limitations with maximum ligand concentration did not allow the calculation of binding stoichiometry. A tendency toward binding was also detected using MST with the $\mathrm{P} 3$ peptide at a maximum concentration of $1,580 \mu \mathrm{M}$ but data quality would not allow a proper fitting for obtaining the $\mathrm{K}_{\mathrm{D}}$ values (Supplementary Figure S4).

\section{DISCUSSION}

Erb1 surface appears to be more suitable for the selection of peptides targeting the complex, as compared to Ytm1 that, despite showing some degree of clustering (Figure 1D) reveals scattered interacting residues and fragmented regions (Supplementary Figure S2). In fact, we did not obtain clear experimental binding results for Ytm1derived peptides. Apart from the peptides derived from Ytm1 selected in this study, additional regions that might have been considered are 224-229 that shows a rather continuous interaction with Erb1. It has, however, been initially discarded given the small differences in calculated $\Delta \Delta \mathrm{G}$ upon in vitro alanine substitution with respect WT Ytm1 in the complex (Supplementary Table S2). An additional region includes V272 that shows reasonable $\Delta \Delta G$ values and continuity (S265-V272) of neighboring residues in Chaetomium. However, it is located in an evolutionary poorly conserved region (Supplementary Figure S2). Sequence elongation of the selected Ytm1-derived peptides might also provide better interaction results.

The combination of in silico hot spot analysis with contact analysis in the interface and residue conservation suggested an 
A

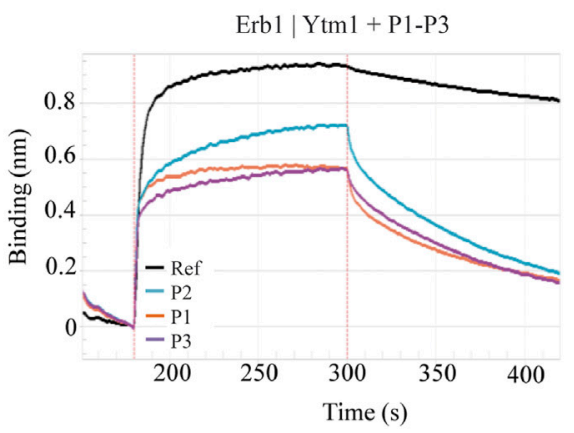

C

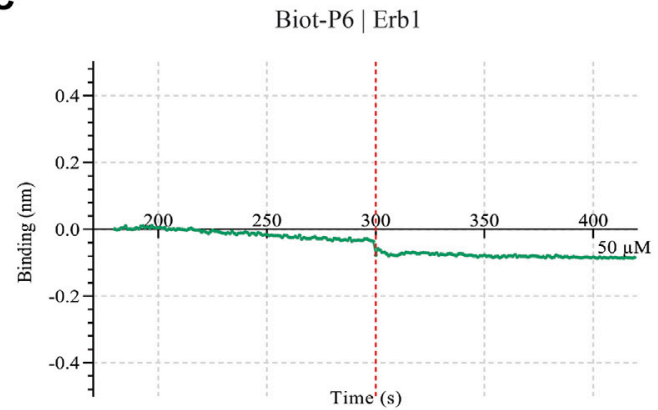

E

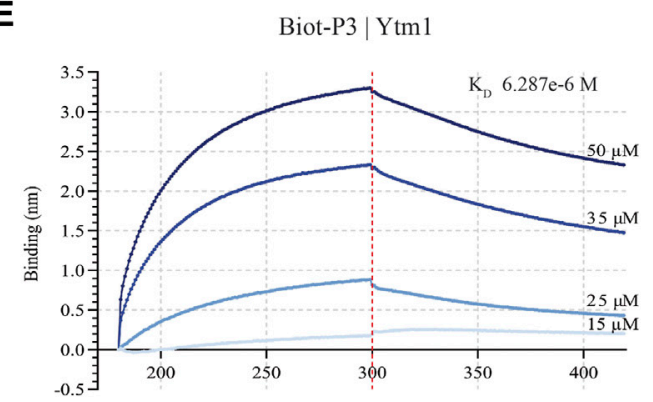

Time (s)

G

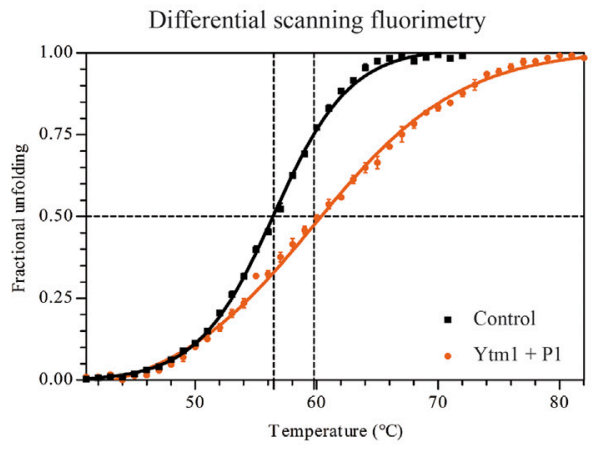

B

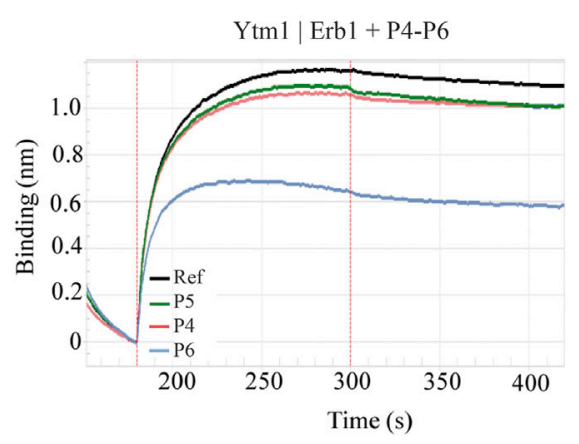

D Biot-P1 | Ytm1

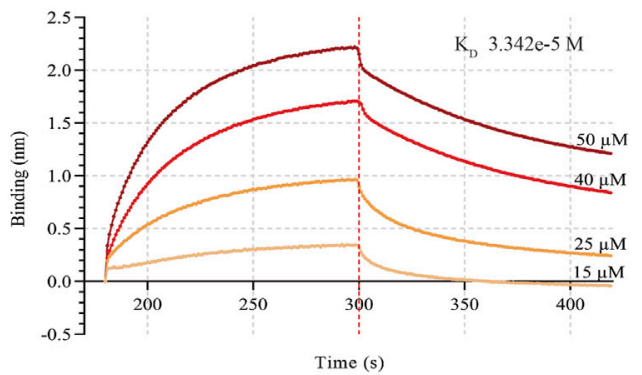

F

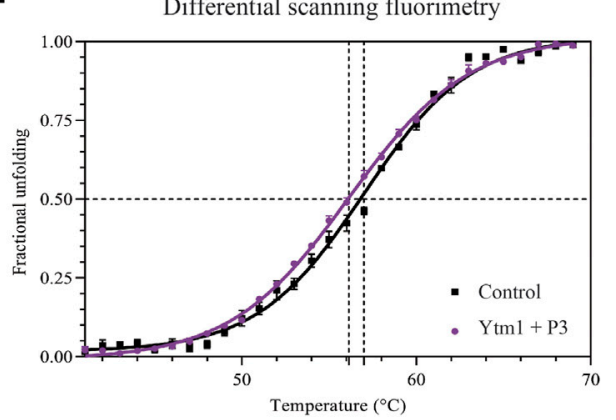

H

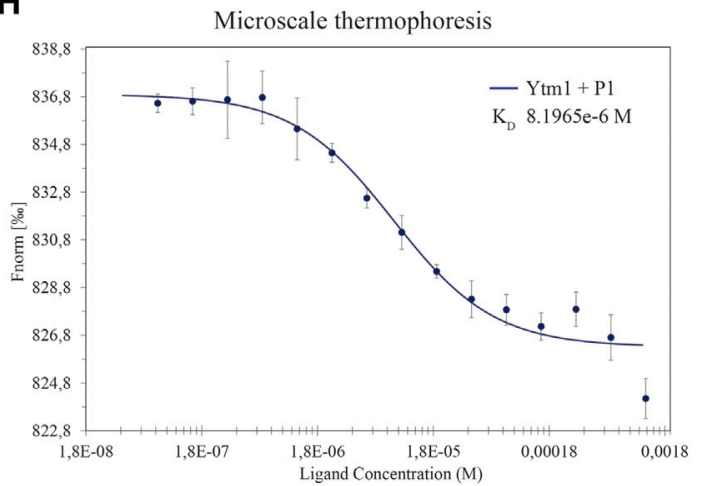

FIGURE 2 | Binding properties of Erb1- and Ytm1-derived peptides to Ytm1 and Erb1, respectively. (A) Biolayer interferometry curves of preincubated peptides P1-3 with equimolar amounts of Ytm1 $(5 \mu \mathrm{M})$. The ability to interfere with Erb1/Ytm1 complex formation (black line) is evaluated using immobilized Erb1 and Ytm1 as an analyte. The reference $K_{D}$ for the Erb1/Ytm1 complex is 3.228e-8M. $K_{D}$ decrease for preincubated Ytm1-P2 is less than one order of magnitude (Supplementary Table S3) whereas it is more than one order of magnitude for preincubated Ytm1-P1 and Ytm1-P3. (B) The equivalent competition BLI experiment preincubating equimolar concentrations of peptides P4-6 with $20 \mu \mathrm{M}$ Erb1 and using immobilized Ytm1 as bait. $K_{D}$ values of Erb1-P5 and Erb1-P4 are similar to the reference $K_{D}$ (2.292e-7M) using only Erb1 as an analyte. $K_{D}$ values of Erb1-P6 are almost one order of magnitude lower than the reference (Supplementary Table S3). (C-E) Biolayer (Continued) 
FIGURE 2 | interferometry curves using inmobilized biotynilated peptides. (C) Immobilized biot-P6 did not show binding when using Erb1 as analyte. (D) BLI Ytm1 binding curves obtained using immobilized biot-P1. (E) BLI Ytm1 binding curves using biot-P3 as bait. (F) Thermal shift curves obtained by differential scanning fluorimetry (DSF) of Ytm1 isolated (black line) and in the presence of $1 \mathrm{mM} \mathrm{P3} \mathrm{peptide.} \mathrm{(G)} \mathrm{Similarly,} \mathrm{DSF} \mathrm{thermal} \mathrm{shift} \mathrm{curves} \mathrm{of} \mathrm{Ytm1} \mathrm{isolated} \mathrm{(black} \mathrm{line)} \mathrm{and} \mathrm{in} \mathrm{the}$ presence of $1 \mathrm{mM} \mathrm{P1}$ peptide (orange). (H) Microscale thermoforesis binding curve for Ytm1 with peptide P1. Ytm1 was labeled using the Red-NHS second-generation dye for the binding affinity with the peptide P1 (NanoTemper Technologies). Fnorm indicates normalized fluorescence.

initial set of six peptides in the Erb1/Ytm1 interface. Three of them showed some degree of interference in the complex formation on a competition assay. Best candidates (P1, P3, and P6) were further tested immobilizing the peptide to the biosensor. We have observed some differences for peptide P6 in the competition assays with respect biotinylated peptide assay using biolayer interferometry. Biotin itself could be affecting the binding to Erb1; however, this is unlikely to be the case since the N-terminus of P6 is not involved in the interaction according to the structure of the Erb1/Ytm1 complex (Figure 1). An obvious difference is the immobilization of the peptide that might be impairing a proper conformation of P6 for the interaction with Erb1 to take place. Immobilized peptide in the biotynilated P1 experiment can also explain the slight difference observed in the Kds obtained by BLI and MST. Tm values for Erb1 using DSF could not be obtained. Moreover, several of the selected peptides did not show any effect on the interference biolayer interferometry assay. Alternative methodological approaches should be considered for the detection of low affinity interactions in BLI or proteins with potential hydrophobic exposed areas in DSF. We have been able to detect direct in vitro interactions of P1 and Biot$\mathrm{P} 1$ with Ytm1 at a low $\mathrm{mM}$ affinity range indicating that at least for these conditions biolayer interferometry is a suitable methodological approach. MST also represented a good alternative for evaluating the interaction with initial binding detected for Ytm1-P3 and proper affinity determination for Ytm1-P1. Erb1 and Ytm1 form a part of the Nop7 complex which is a heterotrimeric complex. Assembly of the three subunits is required for the correct maturation of $60 \mathrm{~S}$ ribosomal subunit. Once the Nop7 complex has exerted its molecular function it is sequentially removed from the pre60S subunit (Tang et al., 2008; Baßler and Hurt, 2019). Individual Nop7, Erb1, and Ytm1 will then reassemble to repeat the process with a new preribosome. It is at this stage when a peptide targeting the Erb1/Ytm1 interaction could play its interference role since the $\mathrm{P} 1$ peptide affinity shown for Ytm1 is lower than the one observed for the Erb1/Ytm1 complex.

Ribosome biogenesis has recently been accumulating growing attention as a potential new therapeutic target, since the observation that cell proliferation can be blocked by inhibition of new ribosomes production. Several RNA pol I inhibitors have been reported to date (Ferreira et al., 2020) and molecules like CX-3543, CX-5461, and $\mathrm{BMH}-21$ are currently under investigation for treating cancer, as rapidly dividing cancer cells are particularly dependent on high levels of RNA pol I transcription. CX-5461 is phase I clinical trial in patients with advanced haematological cancers and breast cancer (Hilton et al., 2018; Khot et al., 2019). CX-3543 reached phase II clinical trial but was withdrawn due to bioavailability issues (Balasubramanian et al., 2011). While extremely promising, these compounds targeting RNA pol I are associated with additional activities, like DNA damage, which possibly contributes to its efficacy, toxicity profile, and resistance mechanisms. These observations suggest that it may be necessary to look for other points of intervention during this multistep process of ribosome maturation. One relevant question in this new field of targeting ribosome biogenesis is whether downstream specific targets would behave the same or differ to those RNA pol I transcription inhibitors currently developed. We had previously shown that altering the interaction surface between the ribosome assembly factors Erb1 and Ytm1 hinders cell proliferation in yeast. We have now developed a peptide derived from the Erb1 sequence capable of interacting with Ytm1 at a low $\mathrm{mM}$ range and interfere in the Erb1/Ytm1 complex formation negatively affecting this extensive and highly conserved protein-protein interaction. This result opens the possibility to investigate the in vivo action of RNA pol I downstream targets in the ribosome biogenesis process. Delivery methods like cell penetrating peptides or nanoparticles should be considered for internalization of the peptide into the cell in order to test future inhibitory strategies in vivo.

\section{DATA AVAILABILITY STATEMENT}

The raw data supporting the conclusions of this article will be made available by the authors, without undue reservation.

\section{AUTHOR CONTRIBUTIONS}

LO-O performed the interaction assays, interaction surface analysis, elaborated tables, and figures, contributed to scientific discussion, and manuscript writing. SM contributed in the peptide selection, interaction surface analysis, provided technical assistance for binding experiments and critical discussion. JB contributed to the peptide selection, methodological approach, graphical imaging, and manuscript writing.

\section{FUNDING}

Funding was obtained from the Spanish Ministry of Economy, Industry and Competitiveness SAF2017-89901-R and Generalitat Valenciana PROMETEO/2018/055. Open access publication fees have been partially funded by URICI from Consejo Superior de Investigaciones Científicas (CSIC). Molecular graphics and analyses were performed with UCSF Chimera, developed by the Resource for Biocomputing, Visualization, and Informatics at the University of California, San Francisco, with support from NIH P41-GM103311.

\section{ACKNOWLEDGMENTS}

We would like to thank Ma del Valle Morales Cuenca, Clara Arenas, and Guilherme Dim for technical support. Molecular 
graphics and analyses were performed with UCSF Chimera, developed by the Resource for Biocomputing, Visualization, and Informatics at the University of California, San Francisco, with support from NIH P41-GM103311 (https://www.cgl.ucsf. edu/chimera/docs/credits.html).

\section{REFERENCES}

Awad, D., Prattes, M., Kofler, L., Rössler, I., Loibl, M., Pertl, M., et al. (2019). Inhibiting Eukaryotic Ribosome Biogenesis. BMC Biol. 17 (1), 1-16. doi:10.1186/s12915-019-0664-2

Balasubramanian, S., Hurley, L. H., and Neidle, S. (2011). Targeting G-Quadruplexes in Gene Promoters: A Novel Anticancer Strategy? Nat. Rev. Drug Discov. 10 (4), 261-275. doi:10.1038/nrd3428

Baßler, J., and Hurt, E. (2019). Eukaryotic Ribosome Assembly. Annu. Rev. Biochem. 88 (1), 281-306. doi:10.1146/annurev-biochem-013118-110817

Bywater, M. J., Poortinga, G., Sanij, E., Hein, N., Peck, A., Cullinane, C., et al. (2012). Inhibition of RNA Polymerase I as a Therapeutic Strategy to Promote Cancer-specific Activation of P53. Cancer Cell 22 (1), 51-65. doi:10.1016/ j.ccr.2012.05.019

Catez, F., Dalla Venezia, N., Marcel, V., Zorbas, C., Lafontaine, D. L. J., Diaz, J.-J., et al. (2019). Ribosome Biogenesis: An Emerging Druggable Pathway for Cancer Therapeutics. Biochem. Pharmacol., 159, 74-81. doi:10.1016/ j.bcp.2018.11.014

Colis, L., Peltonen, K., Sirajuddin, P., Liu, H., Sanders, S., Ernst, G., et al. (2014). DNA Intercalator BMH-21 Inhibits RNA Polymerase I Independent of DNA Damage Response. Oncotarget 5 (12), 4361-4369. doi:10.18632/ oncotarget.2020

Ferreira, R., Schneekloth, J. S., Panov, K. I., Hannan, K. M., and Hannan, R. D. (2020). Targeting the RNA Polymerase I Transcription for Cancer Therapy Comes of Age. Cells 9, 266. doi:10.3390/cells9020266

Granneman, S., Petfalski, E., and Tollervey, D. (2011). A Cluster of Ribosome Synthesis Factors Regulate Pre-RRNA Folding and 5.8S RRNA Maturation by the Rat1 Exonuclease. EMBO J. 30 (19), 4006-4019. doi:10.1038/ emboj.2011.256

Hilton, J., Cescon, D. W., Bedard, P., Ritter, H., Tu, D., Soong, J., et al. (2018). CCTG IND.231: A Phase 1 Trial Evaluating CX-5461 in Patients with Advanced Solid Tumors. Ann. Oncol. 29, iii8. doi:10.1093/annonc/mdy048.003

Khot, A., Brajanovski, N., Cameron, D. P., Hein, N., Maclachlan, K. H., Sanij, E., et al. (2019). First-in-Human RNA Polymerase I Transcription Inhibitor CX5461 in Patients with Advanced Hematologic Cancers: Results of a Phase I Dose-Escalation Study. Cancer Discov. 9 (8), 1036-1049. doi:10.1158/21598290.CD-18-1455

Krissinel, E., and Henrick, K. (2007). Inference of Macromolecular Assemblies from Crystalline State. J. Mol. Biol. 372 (3), 774-797. doi:10.1016/ j.jmb.2007.05.022

Krüger, D. M., and Gohlke, H. (2010). DrugScorePPI Webserver: Fast and Accurate In Silico Alanine Scanning for Scoring Protein-Protein Interactions. Nucleic Acids Res. 38 (Suppl. 2), W480-W486. doi:10.1093/nar/gkq471

Li, Z., Ivanov, A. A., Su, R., Gonzalez-Pecchi, V., Qi, Q., Liu, S., et al. (2017). The OncoPPi Network of Cancer-Focused Protein-Protein Interactions to Inform Biological Insights and Therapeutic Strategies. Nat. Commun. 8, 14356. doi:10.1038/ncomms 14356

Marcin, W., Neira, J. L., and Bravo, J. (2015). The Carboxy-Terminal Domain of Erb1 Is a Seven-Bladed SS-Propeller that Binds RNA. PLoS ONE 10 (4), e0123463. doi:10.1371/journal.pone.0123463

Montanaro, L., Treré, D., and Derenzini, M. (2008). Nucleolus, Ribosomes, and Cancer. Am. J. Pathol. 173, 301-310. doi:10.2353/ajpath.2008.070752

Morcelle, C., Menoyo, S., Morón-Duran, F. D., Tauler, A., Kozma, S. C., Thomas, G., et al. (2019). Oncogenic MYC Induces the Impaired Ribosome Biogenesis

\section{SUPPLEMENTARY MATERIAL}

The Supplementary Material for this article can be found online at: https://www.frontiersin.org/articles/10.3389/fmolb.2021.718941/ full\#supplementary-material

Checkpoint and Stabilizes P53 Independent of Increased Ribosome Content. Cancer Res. 79 (17), 4348-4359. doi:10.1158/0008-5472.CAN-18-2718

Orsolic, I., Jurada, D., Pullen, N., Oren, M., Eliopoulos, A. G., and Volarevic, S. (2016). The Relationship between the Nucleolus and Cancer: Current Evidence and Emerging Paradigms. Semin. Cancer Biol., 37-38, 36-50. doi:10.1016/ j.semcancer.2015.12.004

Pantoliano, M. W., Petrella, E. C., Kwasnoski, J. D., Lobanov, V. S., Myslik, J., Graf, E., et al. (2001). High-Density Miniaturized Thermal Shift Assays as a General Strategy for Drug Discovery. J. Biomol. Scree. 6 (6), 429-440. doi:10.1177/ 108705710100600609

Pettersen, E. F., Goddard, T. D., Huang, C. C., Couch, G. S., Greenblatt, D. M., Elaine, C. M., et al. (2004). UCSF Chimera?A Visualization System for Exploratory Research and Analysis. J. Comput. Chem. 25 (13), 1605-1612. doi:10.1002/jcc.20084

Studier, F. W. (2005). Protein Production by Auto-Induction in High-Density Shaking Cultures. Protein Expr. Purif. 41 (1), 207-234. doi:10.1016/ j.pep.2005.01.016

Tang, L., Sahasranaman, A., Jakovljevic, J., Schleifman, E., and Woolford, J. L. (2008). Interactions Among Ytm1, Erb1, and Nop7 Required for Assembly of the Nop7-Subcomplex in Yeast Preribosomes. Mol. Biol. Cel 19 (7), 2844-2856. doi:10.1091/mbc.E07-12-1281

Truitt, M. L., and Ruggero, D. (2016). New Frontiers in Translational Control of the Cancer Genome. Nat. Rev. Cancer 16, 288-304. doi:10.1038/nrc.2016.27

Volarevic, S., Stewart, M. J., Ledermann, B., Zilberman, F., Terracciano, L., Montini, E., et al. (2000). Proliferation, but Not Growth, Blocked by Conditional Deletion of 40S Ribosomal Protein S6. Science 288 (5473), 2045-2047. doi:10.1126/science.288.5473.2045

Wegrecki, M., Rodríguez-Galán, O., de la Cruz, J., and Bravo, J. (2015). The Structure of Erb1-Ytm1 Complex Reveals the Functional Importance of a HighAffinity Binding between Two $\beta$-Propellers During the Assembly of Large Ribosomal Subunits in Eukaryotes. Nucleic Acids Res. 43 (22), 11017-11030. doi:10.1093/nar/gkv1043

Winn, M. D., Ballard, C. C., Cowtan, K. D., Eleanor, J. D., Paul, E., Dodson, E. J., et al. (2011). Overview of theCCP4 Suite and Current Developments. Acta Crystallogr. D Biol. Cryst. 67, 235-242. doi:10.1107/S0907444910045749

Zhao, Y., Chapman, D. A. G., and Jones, I. M. (2003). Improving Baculovirus Recombination. Nucleic Acids Res. 31 (2), E6. doi:10.1093/nar/gng006

Conflict of Interest: The authors declare that the research was conducted in the absence of any commercial or financial relationships that could be construed as a potential conflict of interest.

Publisher's Note: All claims expressed in this article are solely those of the authors and do not necessarily represent those of their affiliated organizations, or those of the publisher, the editors and the reviewers. Any product that may be evaluated in this article, or claim that may be made by its manufacturer, is not guaranteed or endorsed by the publisher.

Copyright $\odot 2021$ Orea-Ordóñez, Masiá and Bravo. This is an open-access article distributed under the terms of the Creative Commons Attribution License (CC BY). The use, distribution or reproduction in other forums is permitted, provided the original author(s) and the copyright owner(s) are credited and that the original publication in this journal is cited, in accordance with accepted academic practice. No use, distribution or reproduction is permitted which does not comply with these terms. 